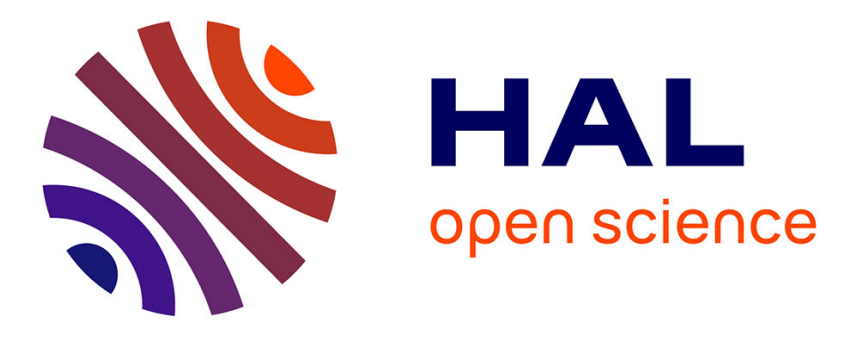

\title{
Automatic model generation framework for computational simulation of cochlear implantation
}

Nerea Mangado, Mario Ceresa, Nicolas Duchateau, Hans Martin Kjer, Sergio

Vera, Hector Dejea Velardo, Pavel Mistrik, Rasmus R. Paulsen, Jens

Fagertun, Jérôme Noailly, et al.

\section{To cite this version:}

Nerea Mangado, Mario Ceresa, Nicolas Duchateau, Hans Martin Kjer, Sergio Vera, et al.. Automatic model generation framework for computational simulation of cochlear implantation. Annals of Biomedical Engineering, 2016, 44 (8), pp.2453-2463. 10.1007/s10439-015-1541-y · hal-01314765

\section{HAL Id: hal-01314765 \\ https://hal.inria.fr/hal-01314765}

Submitted on 12 May 2016

HAL is a multi-disciplinary open access archive for the deposit and dissemination of scientific research documents, whether they are published or not. The documents may come from teaching and research institutions in France or abroad, or from public or private research centers.
L'archive ouverte pluridisciplinaire HAL, est destinée au dépôt et à la diffusion de documents scientifiques de niveau recherche, publiés ou non, émanant des établissements d'enseignement et de recherche français ou étrangers, des laboratoires publics ou privés. 


\title{
Automatic model generation framework for computational simulation of cochlear implantation
}

\author{
Nerea Mangado $^{1}$, Mario Ceresa ${ }^{1}$, Nicolas Duchateau ${ }^{2}$, Hans Martin \\ Kjer $^{3}$, Sergio Vera ${ }^{4}$, Hector Dejea ${ }^{1}$, Pavel Mistrik ${ }^{5}$, Rasmus R. \\ Paulsen $^{3}$, Jens Fagertun ${ }^{3}$, Jérôme Noailly ${ }^{1}$, Gemma Piella ${ }^{1}$, and \\ Miguel Ángel González Ballester ${ }^{1,6}$ \\ ${ }^{1}$ Simbiosys group, Universitat Pompeu Fabra, Barcelona, Spain \\ ${ }^{2}$ Asclepios Research project, INRIA Sophia Antipolis, France \\ ${ }^{3}$ Denmark Technical University, Copenhagen, Denmark \\ ${ }^{4}$ Alma Medical Imaging, Barcelona, Spain \\ ${ }^{5}$ Med-EL, Innsbruck, Austria \\ ${ }^{6}$ ICREA, Barcelona, Spain
}

Address of correspondence to Nerea Mangado, Department of Information and Communication Technologies, Universitat Pompeu Fabra, Tànger Building, 08018, Barcelona, Spain. Electronic mail: nerea.mangado@upf.edu

\begin{abstract}
Recent developments in computational modeling of cochlear implantation are promising to study in-silico the performance of the implant before surgery. However, creating a complete computational model of the patient's anatomy while including an external device geometry remains challenging. To address such a challenge, we propose an automatic framework for the generation of patient-specific meshes for finite element modeling of the implanted cochlea. First, a statistical shape model is constructed from highresolution anatomical $\mu \mathrm{CT}$ images. Then, by fitting the statistical model to a patient's CT image, an accurate model of the patient-specific cochlea anatomy is obtained. An algorithm based on the parallel transport frame is employed to perform the virtual insertion of the cochlear implant. Our automatic framework also incorporates the surrounding bone and nerve fibers and assigns constitutive parameters to all components of the finite element model. This model can then be used to study in-silico the effects of the electrical stimulation of the cochlear implant. Results are shown on a total of 25 models of patients. In all cases, a final mesh suitable for finite element
\end{abstract}


simulations was obtained, in an average time of 94 seconds. The framework has proven to be fast and robust, and is promising for a detailed prognosis of the cochlear implantation surgery.

Keywords: automatic framework, three dimensional finite element mesh, statistical shape model, cochlear implants, multi-object modeling, virtual surgical insertion.

\section{Introduction}

Sensorineural hearing loss is a common cause of disability. According to the World Health Organization, $27 \%$ of men and $24 \%$ of women above 45 years of age suffer from more than $26 \mathrm{~dB}$ hearing loss. Cochlear implantation (CI) is a surgical procedure whereby an electronic device replaces the hearing system. This device directly stimulates the auditory nerves via an electrode array (EA) placed in the scala tympani of the cochlea. However, the outcome of this technique highly depends on patient-specific factors and, as a consequence, the level of hearing restoration can substantially vary [16]. We believe that computational modeling is useful to simulate different implantation scenarios of a given patient, and, moreover, can improve the selection of the surgical procedure parameters.

Personalization of computational models has a huge potential to evaluate insilico the effect of patient-specific factors, since it allows parametric predictions of treatment outcome and patient-specific treatment optimization [26]. In particular, an individualized model would play a decisive role in the cochlear implantation to choose the implant design that better fits the patient's diagnosis. To this end, a combined computational model of both patient's inner ear anatomy and implant is needed.

Recently, the generation of computational finite element models from biomedical data has been widely studied [35, 15]. The mesh generation of structures extracted from medical imaging is a common practice in biomedical computational applications [36, 28, 21, 11]. However, some complications have been identified, such as the presence of holes or the low quality of the triangulation [28, 23]. Capturing the anatomy of the patient is of prime importance, but modeling the implant according to the patient's anatomy is not straightforward, as pointed out in the exploration of stent designs for intracranial aneurysms [22] or in the case of transcatheter aortic valve designs [32]. In these cases, the deformation of a stent geometry design was carried out to physically fit the patient's anatomy by reorienting the mesh according to the internal and external forces or forcing the displacement of the nodes towards a defined centerline [22, 32].

In the case of cochlear implants, an EA needs to be inserted into the cochlea during the surgery. Thus, the EA computational model needs to be adapted to the 
anatomy of the patient. Previous attempts used a simplified approach, generating an extrusion of the cross-section of the cochlear anatomy and the implant along a spiral in three dimensions $[17,31,3]$. Another approach to model the EA and its insertion consists in considering a friction model, in which forces and contact pressures are computed at each step of the implant insertion [39, 7]. However, these approaches do not adapt the implant to a three dimensional cochlear anatomy. In fact, the insertion of the EA highly contributes to the variability in CI outcomes $[37,14,12]$. For this reason, we do believe that the electrode insertion needs to be accurately defined in a CI computational model.

The approach we introduce allows for an automatic adaptation of the EA according to a patient-specific anatomy. We have already proposed a framework for patient-specific electrical simulation after CI. This approach provides useful information for surgical planning of the CI, by performing in-silico analysis of implant placement and function before surgery $[5,24,6]$. However, the computational model was created in a non-automatic way and the deformation of the implant was manually performed, which was neither accurate nor adequate for systematic explorations of personalized solutions. Some of the limitations of the virtual insertion were already addressed, which partially improved the personalization of CI computational studies $[9,25]$.

In this work, we present a completely automatic mesh generation pipeline for the creation of computational models of CI surgery. The framework proposed includes (1) the generation of a cochlear surface obtained from a statistical shape model (Section 2.1), (2) the virtual insertion that performs a patient-specific placement of the EA (Section 2.2.2), (3) the creation of the auditory nerve fibers of the inner ear (Section 2.3) and (4) the generation of a mesh to carry out finite element (FE) simulations (Section 2.4) (see Figure 1). The ultimate goal of this framework is to study in depth the effect of the implant placement and function on each patient. This will help to determine the best parameters of the implantation, in terms of geometric design and electrical stimulation protocol.

\section{Materials and Methods}

\subsection{Statistical Shape Model construction}

Modeling of CI is often approached by simplified geometric models, or a single model extracted from imaging data of one patient. However, these approaches do not consider the variability found among patients. For this reason, the current work considered the creation of a statistical shape model (SSM). Based on Cootes' work [8], a SSM is trained from a set of example shapes. Each shape is represented by a set of points in a common coordinates system. The average shape can be computed 


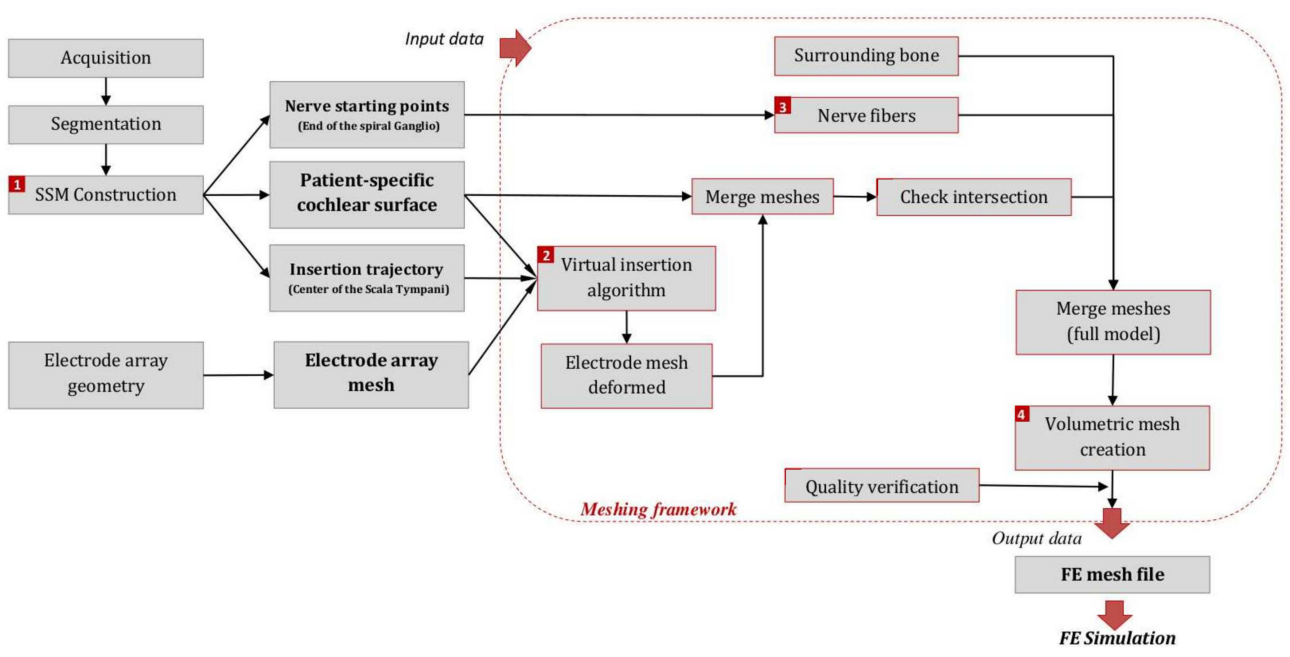

Figure 1: Pipeline of the automatic mesh generation framework. A patient-specific shape is estimated based on the statistical shape model (1), a virtual insertion of the EA is performed (2), the surrounding bone and nerves are generated (3). Then all meshes are merged into a single triangular mesh which, after checking for intersections and mesh quality, is then converted into a volumetric mesh of the whole model (4). Finally, the mesh is converted to a suitable file for finite element simulation.

by simply averaging the corresponding landmarks, and a principal component analysis provides the main modes of shape variation. Through this process, a compact representation of low dimensionality is obtained, which encodes the shape variability as a small set of scalar weights modulating the contribution of each of the modes of variation.

A SSM was built from 17 samples of the inner ear from human cadavers, scanned with a high resolution $\mu \mathrm{CT}[5]$. These data were manually segmented and the surface model of the inner ear extracted. The surfaces were aligned to a reference shape, chosen from the training dataset, by a non-rigid registration method based on multi-level B-splines [20]. Principal component analysis was then employed to build the SSM. The SSM can serve as a generative model, allowing to sample plausible anatomical shapes of the population by varying the weights associated to the main modes of variation (Fig. 2). By constraining the weights of the SSM, we avoid applying a high deformation that could create a highly deformed and unrealistic shape, which can lead to an undesired cochlear surface model. Thus, we obtain realistic representations of the cochlea anatomy that can be found among the modeled population. 


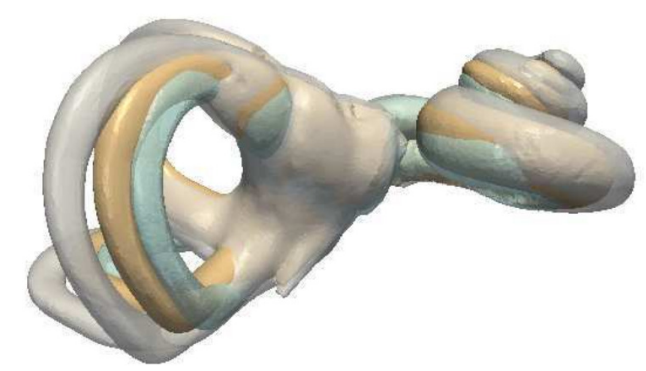

Figure 2: Statistical Shape Model. Mean shape (beige) and shapes generated by applying \pm 1 standard deviation of the first mode of variation (blue and grey surfaces).

Secondly, the SSM can be used as a statistical shape prior for image registration between a clinical CT dataset of low resolution (from 100 to $400 \mu \mathrm{m}$ ) and a highresolution $\mu \mathrm{CT}(25 \mu \mathrm{m})$ instance of the SSM. This allows making a patient specific prediction of the detailed anatomical shape from clinical CT, without the need to perform a manual segmentation [19]. As a result, we obtain a high-resolution surface mesh representing the cochlear shape of the patient (Fig. 1, Step 1).

\section{$2.2 \quad$ Virtual insertion}

Once the surface of the patient's anatomy is obtained, the implant is virtually placed (Fig. 1, Step 2). The objective is to deform the implant mesh according to the cochlear anatomy of the patient, simulating the surgical insertion. Previously, authors evaluated the contact pressures that the EA causes to the lateral wall of the cochlea by computing the deformation on the implant $[39,7]$. Others created a geometrical extrusion of the implant following a spiral similar to the cochlear structure $[17,3]$. In this work, we aim to perform a physical simulation of the insertion and adapt the original model of the commercial implant to the 3D model of the patient inner ear [9], leading to a more detailed and realistic model. Our simulation framework consists of two steps. First, a process of insertion of the electrode array was simulated using a simplified electrode mesh. This step-by-step simulation provided the EA position inside the cochlea. It was complex and timeconsuming, but only needed to be performed once, on the shape used as reference in the SSM. The insertion path obtained was then stored and propagated as part of the SSM fitting, to generate a path for the patient-specific mesh. In a second step, the detailed EA mesh was adapted to the patient mesh, following the specified insertion parameters. 


\subsubsection{Simulation of surgical insertion of the electrode array}

A mechanical simulation of the insertion provides the EA trajectory. Our approach was based on the open source simulation framework SOFA [1]. The virtual placement was modeled as a scene graph including the objects and their physical properties. The cochlear geometry, obtained from the SSM fitting, was defined in the scene as a fixed rigid object and the electrode as an elastic object simulated with a linear FE method-based simulator [27] and with the following material properties: $470 \mathrm{MPa}$ and 0.4 for the Young modulus and Poisson ratio, respectively. Before the simulation, preprocessing discarded most of the vestibule and the semicircular canals, as the insertion needed to consider exclusively the cochlear area. Additionally, a hole was generated at the round window niche, where the electrode was inserted during surgery. The electrode behavior was determined by three components:

(a) The collision model, which keeps track of the interactions between the electrode and the cochlear mesh.

(b) The tetrahedral mesh for the FE model simulation, which consists of a FE model with less elements for fast simulation, around 5-10 minutes compared to the original mesh, which takes 40 minutes.

(c) The triangular mesh for visual representation, which follows the real EA specifications.

The simulation tracked the three individual components and applied the collision detection to the collision model, integrating forces to the tetrahedral mesh and drawing the visual representation (Figure 3A). A set of mapping algorithms kept the coherency between the different models. An additional object simulated the insertion guide used by the surgeon to reach the cochlea. This object consisted in a hollow collision cylinder, and prevented the electrode from bending out of shape while the applied force inserted it into the cochlea. The electrode was introduced through the round window niche, and the simulation stopped the insertion according to a specified maximal insertion depth. The position and the direction of the electrode tip were evaluated at each integration step ensuring the tangential direction of the electrode. This surgical simulation resulted in the insertion trajectory of the EA and needed to be performed only once, off-line, on the SSM reference shape. It was then adapted automatically to the specific cochlear anatomy as part of the SSM fitting process.

\subsubsection{Virtual EA placement via parallel transport frame}

The insertion of the detailed EA mesh geometry is guided through the geometrical matching of the electrode centerline and the insertion trajectory curve, ob- 
tained as described above. Classical parametrization frames, such as the tangential/normal/binormal frame, are highly sensitive to curvature changes along the centerline, which may lead to artifacts $[9,2]$. Consider a 3D curve parametrized by its length:

$$
\mathbf{x}(s) \mid s \in[0,1], \mathbf{x} \in \mathbb{R}^{3} .
$$

A local parametrization based on the parallel transport of a given orthonormal basis $\left(\mathbf{T}(0), \mathbf{E}_{\mathbf{1}}(0), \mathbf{E}_{\mathbf{2}}(0)\right)$ along this curve is defined as [2]:

$$
\left[\begin{array}{c}
\mathbf{T}^{\prime}(s) \\
\mathbf{E}_{\mathbf{1}}{ }^{\prime}(s) \\
\mathbf{E}_{\mathbf{2}}{ }^{\prime}(s)
\end{array}\right]=\left[\begin{array}{ccc}
0 & k_{1}(s) & k_{2}(s) \\
-k_{1}(s) & 0 & 0 \\
-k_{2}(s) & 0 & 0
\end{array}\right]\left[\begin{array}{c}
\mathbf{T}(s) \\
\mathbf{E}_{\mathbf{1}}(s) \\
\mathbf{E}_{\mathbf{2}}(s)
\end{array}\right],
$$

where $k_{1}$ and $k_{2}$ are the components of the curvature vector with respect to the basis, and:

$$
\begin{aligned}
& \mathbf{E}_{\mathbf{1}}(s)=\mathbf{r}(s)-\langle\mathbf{r}(s), \mathbf{T}(s)\rangle \mathbf{T}(s) \\
& \mathbf{E}_{\mathbf{2}}(s)=\mathbf{T}(s) \times \mathbf{E}_{\mathbf{1}}(s)
\end{aligned}
$$

where $\mathbf{r}=\mathbf{R} \cdot \mathbf{E}_{\mathbf{1}}(s-d s)$, ds is an infinitesimal element of length and $\mathbf{R}$ the $3 \mathrm{D}$ rotation matrix encoding the change of local basis.

The virtual insertion starts by matching the centerline of the EA to the trajectory insertion curve. Then, for each node $\mathbf{p}$ of the electrode mesh, the closest

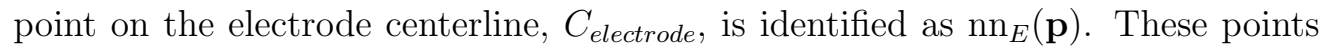
are defined by a local $3 \mathrm{D}$ vector in the $\left(\mathbf{e}_{x}, \mathbf{e}_{y}, \mathbf{e}_{z}\right)$ basis:

$$
\mathbf{v}_{E}(\mathbf{p})=\left[\begin{array}{l}
v_{E, x} \\
v_{E, y} \\
v_{E, z}
\end{array}\right]_{\left(\mathbf{e}_{x}, \mathbf{e}_{y}, \mathbf{e}_{z}\right)}=\mathbf{p}-\mathrm{nn}_{E}(\mathbf{p}) .
$$

The same decomposition is used in the cochlear trajectory curve coordinates:

$$
\mathbf{v}_{C}(\mathbf{p})=\left[\begin{array}{l}
v_{E, x} \\
v_{E, y} \\
v_{E, z}
\end{array}\right]_{\left(\mathbf{E}_{\mathbf{2}}, \mathbf{T}, \mathbf{E}_{\mathbf{1}}\right)}
$$

Finally, the points of the reoriented electrode are reconstructed as

$$
\mathbf{p}_{\text {reoriented }}=\mathrm{nn}_{C}(\mathbf{p})+\mathbf{v}_{C}(\mathbf{p})
$$

where $\mathbf{n n}_{C}$ is the cochlear mesh. Figure 3 shows an example of the results of the virtual insertion algorithm. 
This a pre-print version.

The final document is available at http://www.springerlink.com
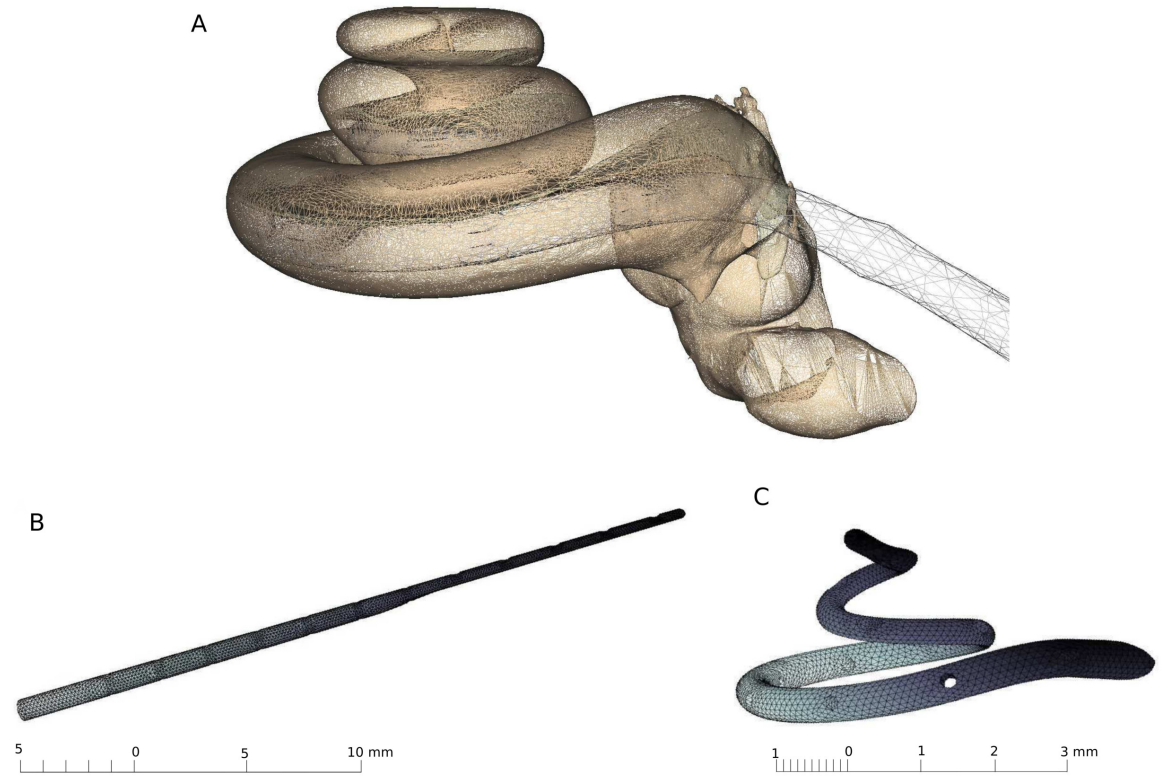

Figure 3: Virtual insertion. (A) Surgical insertion simulation by SOFA. The position during the insertion of the electrode array into the cochlea is displayed. Virtual placement of the EA mesh before (B) and after (C) the reorientation via parallel transport frame. 
Depth of insertion can significantly influence the outcome of the surgery [13]. Our approach controls the initial orientation and depth of the insertion, by respectively setting the initial basis $\left(\mathbf{T}(\mathbf{0}), \mathbf{E}_{\mathbf{1}}(\mathbf{0}), \mathbf{E}_{\mathbf{2}}(\mathbf{0})\right)$ and determining the length of the centerline portion where insertion happens. Partials insertions with 1, 2 or 3 contacts of the EA outside the cochlea can be achieved. Such partial insertions may result from a shape mismatch between the cochlear implant electrode chosen by the clinician and the patient's cochlear anatomy. The mismatch refers to an EA longer/shorter than that which would be adequate to the patient's cochlea dimensions. Thus, if the EA is longer, part of the electrode array would be outside the cochlea during the intervention. This can be prevented by using in-silico modeling in CI, which allows planning the intervention virtually, prior to the actual surgery.

The EA design used in the reported experiments is based on the Flex28 model of the manufacturer Med-EL GmbH (Innsbruck, Austria). Its geometrical design is defined by a length of $28 \mathrm{~mm}$ and an initial diameter of $0.8 \mathrm{~mm}$. It has 19 electrode contacts spaced within a $23.1 \mathrm{~mm}$ region, called the active stimulation range of the EA (Figure 3B). The synthetic CAD geometry of the EA was created with Gmesh, obtaining a triangular surface of approximately 10.000 elements. A linear heat diffusion process was carried out along the length of the electrode array to parametrize its geometry. This provides a series of points that form the electrode centerline. This line is used to match, by the parallel transport frame approach, the electrode array to the trajectory curve of the surgical virtual insertion obtained with SOFA.

\subsection{Nerve fibers}

Including the nerve fibers into the computational model allows exploring the activation patterns caused by the electrical stimulation of the cochlear implant. Three coordinates from three different sets of points were used to reconstruct each nerve fiber. The first set of points belong to a trajectory curve manually extracted from the reference shape surface of the SSM (see Figure 4A). The trajectory curve was located at the transition of the Organ of Corti and the beginning of the basilar membrane along the cochlea. Each nerve fibre started from one of the points of this curve (Figure 1, Step 3). This process was performed only once, off-line, in the reference mesh. We chose this approach to propagate the information automatically to patient-specific shapes via SSM fitting. Thus, no further manual extraction of data is needed. When a new virtual patient is generated, the deformation can be applied to the trajectory curve. Then, it will be in correspondence with the geometry of the virtual patient sampled.

The trajectory curve was projected into a 2D plane through an eigenanalysis and the center of mass is computed. The coordinates of this center of mass were used as reference target for the end of the nerve fiber. For each nerve, a third middle 
point is obtained by scaling down the previously projected line in the $2 \mathrm{D}$ plane. Finally, the three coordinate points of each nerve fiber are obtained by recovering the third dimension of the original trajectory curve (Figure 4A). A parametric spline interpolation is done to obtain a smooth curve representing the whole nerve fiber. We chose a B-spline formulation in order to keep the generality of the approach and be able to easily increase the number of points. Points sampled along the fiber are defined as nerve compartments, where the electrical potential due to the cochlear implant stimulation will be obtained through further FE simulations [6]. The number of nerves to be created was defined as an input variable. From our experience, 100 nerve fibers is an affordable compromise to guarantee a proper resolution, while not affecting performance. Figure 4B shows an example of nerve fibers computed in a cochlear shape sampled from the SSM.
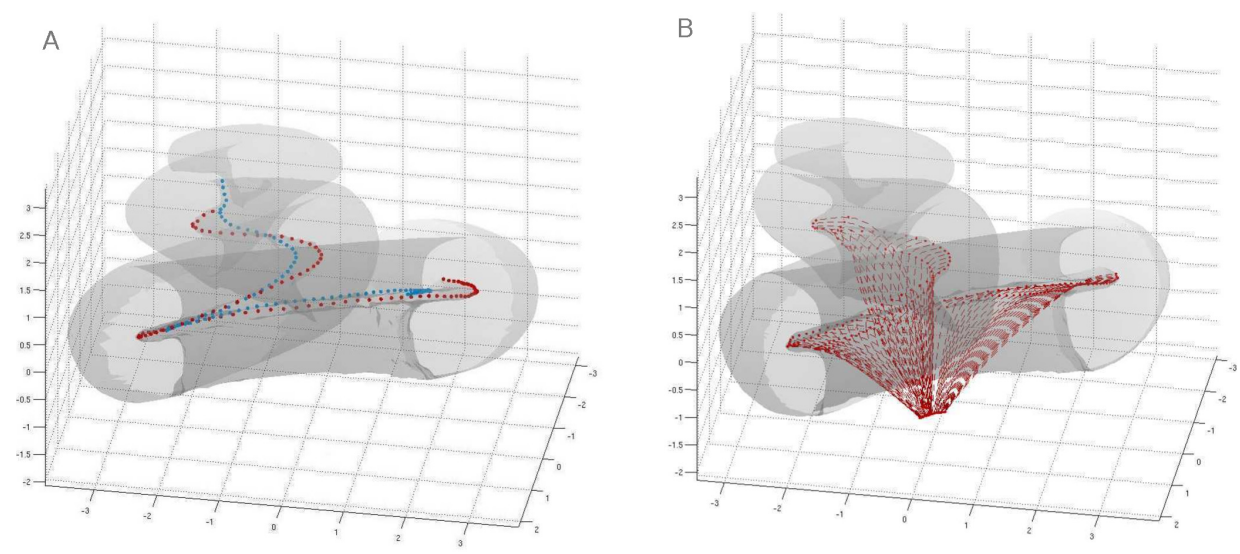

Figure 4: Nerve fiber creation. (A) Red: Trajectory line at the Organ of Corti. Blue: Middle coordinates of each nerve fiber. (B) Nerve fibers created.

\subsection{Mesh generation}

This step involves the creation of the whole mesh combining the patient-specific cochlear shape, the EA geometry adapted to the patient, the nerve fibers, and the surrounding bone. Our meshing framework is based on the Matlab open-source processing toolbox Iso2mesh [11], focused mainly on creating and processing FE meshes from surfaces and volumetric medical images. The cochlear surface is remeshed through conversion into a volumetric binary image and transformed into a finer surface mesh, which guarantees the lack of self-intersecting elements [11]. 
The volumetric size of the cochlear model is computed, and an outer box mesh is created, representing the surrounding bone around the cochlea.

Intersections between the cochlear surface and the implant occur in cases with partial insertion, in which the beginning of the EA is located in the outer part of the cochlea. Usually, the surgeon wraps this external part and leaves it outside, since it does not cause damage to the patient. However, simulating this situation is not feasible, since penetrations of the EA mesh elements into the cochlear mesh would cause errors in the further modeling and simulation steps (Figure 1, Step 4). To prevent these errors, a sphere is generated at the basal part of the cochlea, and the intersection between the implant and sphere meshes is computed. Both the sphere and the outer part of the implant are removed, keeping exclusively the range of the implant mesh inside the cochlea. Due to the intersection plane created in this process, the newly generated elements may have poor quality (see Figure 5). This is solved by re-meshing the implant.
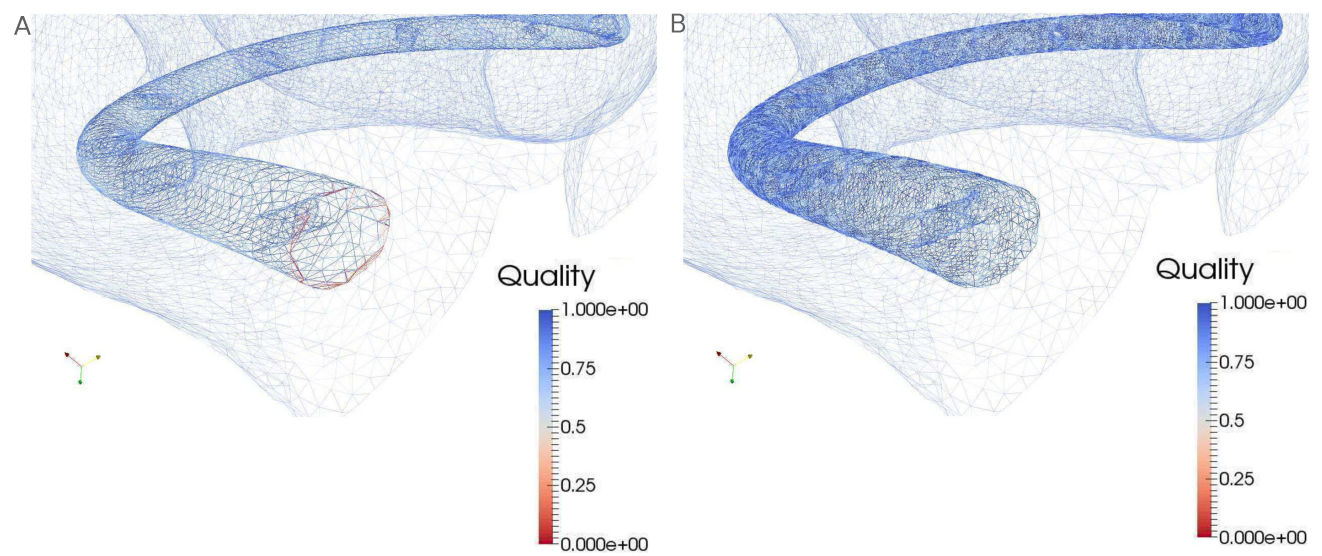

Figure 5: Solving low quality elements. Before (A) and after (B) the remeshing of the implant mesh to improve the mesh quality. In this case, element quality has been assessed by their aspect ratio [34].

All meshes are then merged into a single combined model by means of the Iso2mesh toolbox. An automatic final check for possible intersections is performed [11]. Finally, the empty space inside the triangular mesh is filled in, creating new volumetric elements. The result is a tetrahedral mesh of the whole model.

\subsubsection{Material properties and boundary conditions}

A label is automatically created for each body of the final tetrahedral mesh: a total of 15 bodies, where 12 of them correspond to the electrodes and the rest to 
Table 1: Material Properties

\begin{tabular}{|c|c|c|c|c|}
\hline Material & Cochlea & Bone & Silicone & Electrode \\
\hline $\begin{array}{c}\text { Resistivity } \\
(\Omega \cdot m)\end{array}$ & 0.70 & 6.41 & 10.1 & 0.001 \\
\hline
\end{tabular}

the cochlea, bone and electrode array. These labels are assigned to the elements that form each of the previous bodies. This allows defining the material properties of each of them, according to [5] (see Table 1).

In the same way, electrical boundary conditions are defined according to the stimulation protocol, that defines the activation of the electrodes, and the parameters to be evaluated, such as the current delivered by the implant. Both material properties and boundary conditions are set in the reference model of the SSM, and are then automatically propagated to the patient-specific model. This generates a complete computational model file ready for FE simulation (Figure 8). In order to ensure a proper geometrical aspect of the mesh elements, which may influence in further FE simulations, the mesh quality is verified (Fig.1, Step 5) by the following interpolation quality measure for tetrahedral elements [34]:

$$
\mathbf{Q}_{\text {tet }}=3^{\frac{7}{4}} \frac{V}{\left(\sum_{i=1}^{4} A_{i}^{2}\right)^{\left(\frac{3}{4}\right)}}
$$

where $A_{i}$ and $V$ stand for the face area and volume of the element. This measure takes value between 0 and 1 , for nearly degenerated and regular tetrahedral element, respectively.

\section{Results}

The framework was tested on 25 cochlear shapes generated randomly from the SSM. The average trajectory curve obtained from these shapes had a length of $20.84 \pm 1.64 \mathrm{~mm}$ and a number of turns of $1.80 \pm 0.08$. In all cases, the framework was fully automatic and led to a final mesh of the whole CI model in a proper format for a FE solver.

The EA insertion was classified as full or partial insertion. Virtual full insertion was in average $18.4 \pm 1.1 \mathrm{~mm}$. Changes in the electrode mesh size were analyzed. The area of each mesh element were compared (see Figure 6A). The average reduction of element area was $-25.0 \pm 0.3 \%$. Figure $6 \mathrm{~B}$ illustrates the local change of each element area on one case. Different virtual insertions were tested to prove the sensitivity of the algorithm to the parameters.

Partial insertions were simulated from a range spanning from full insertion to 3 contacts out, meaning for this specific model 23 and $18 \mathrm{~mm}$. An example of the 
parallel transport procedure is shown in Figure 7. It depicts full insertion, one, two and three contacts out. On average, a model generation took $94 \pm 11 \mathrm{~s}$ for the complete meshing framework (see Figure 1). The final mesh quality was 0.8 \pm 0.15 according to the metric defined in equation 6 .
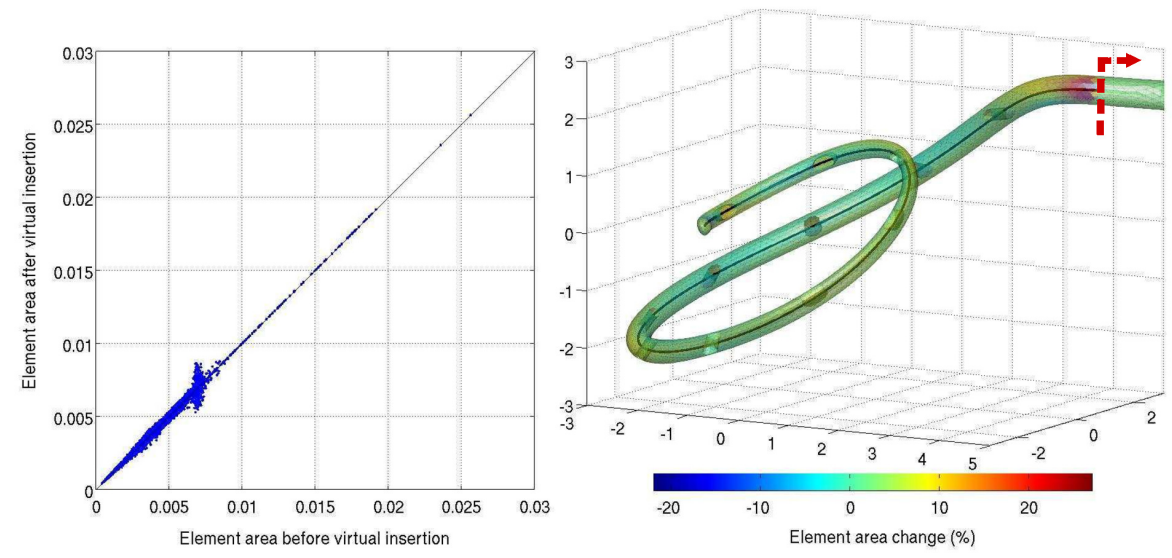

Figure 6: Changes in the EA geometry. (A) Area of each triangle of the electrode surface mesh before and after the virtual insertion. (B) Changes in the element geometry after deformation (\%) compared to the area before the virtual insertion. The dashed red line delimits the part of the electrode that remains outside the cochlea.

\section{Discussion and Conclusions}

We presented an automatic framework for the generation of personalized FE meshes to be used in CI computational models. A SSM is built from high-resolution $\mu \mathrm{CT}$ scans, and used to generate detailed models, either via SSM sampling or by fitting it to a low-resolution clinical CT image. The method includes virtual implant placement, realistic both in terms of mechanical interactions and geometric representation of the EA. It can merge heterogeneous meshes, obtained from images, CAD design and synthetic data. The framework seamlessly combines them into a FE mesh, ensuring its quality in terms of element shape and lack of intersection. This patient-specific model is ready to be used for computational simulations. Moreover, it allows in silico studies of parameters that cannot be measured directly in clinical practice.

Different workflows to generate FE meshes for biomedical models exist in the literature $[33,4,38]$. However, none of these approaches were automatic or took 


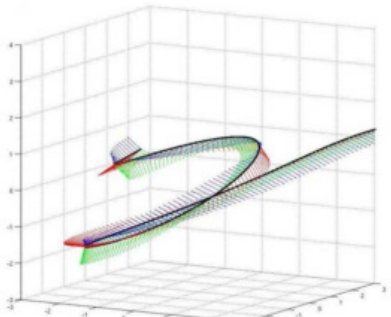

Full insertion

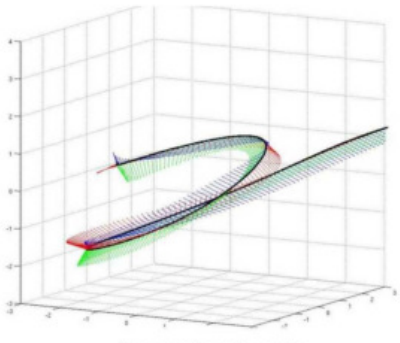

1 contact out

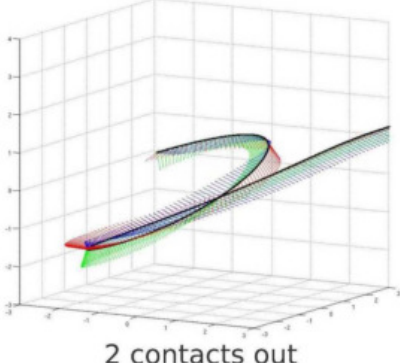

2 contacts out

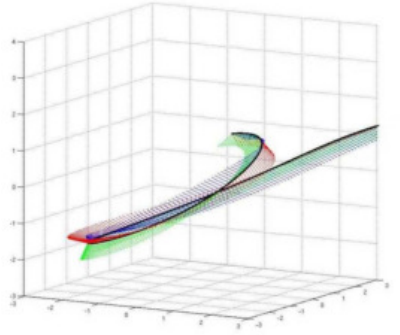

3 contacts out

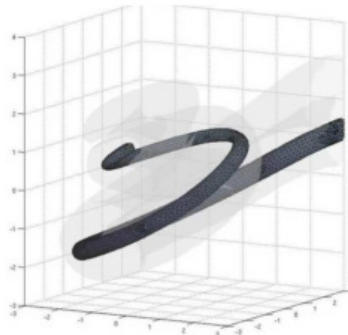

Full insertion

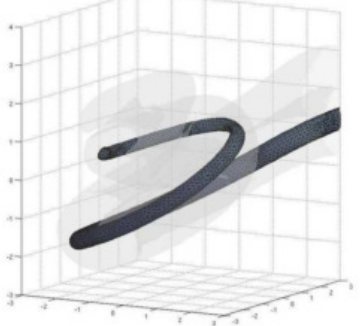

1 contact out

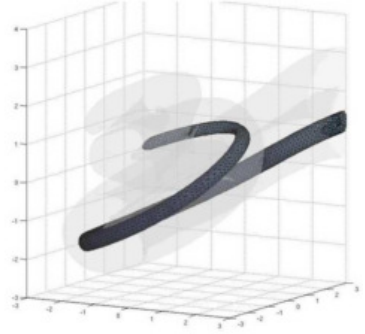

2 contacts out

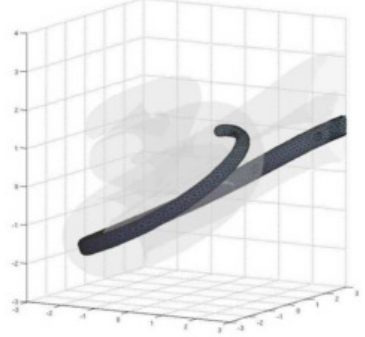

3 contacts out

Figure 7: Virtual electrode placement for full insertion, one, two and three contacts outside the cochlea. Left: vector representation of the parallel transport frame (orthogonal basis) along the entire cochlea trajectory curve. Right: Output of the virtual insertion of the EA mesh into the cochlea. 


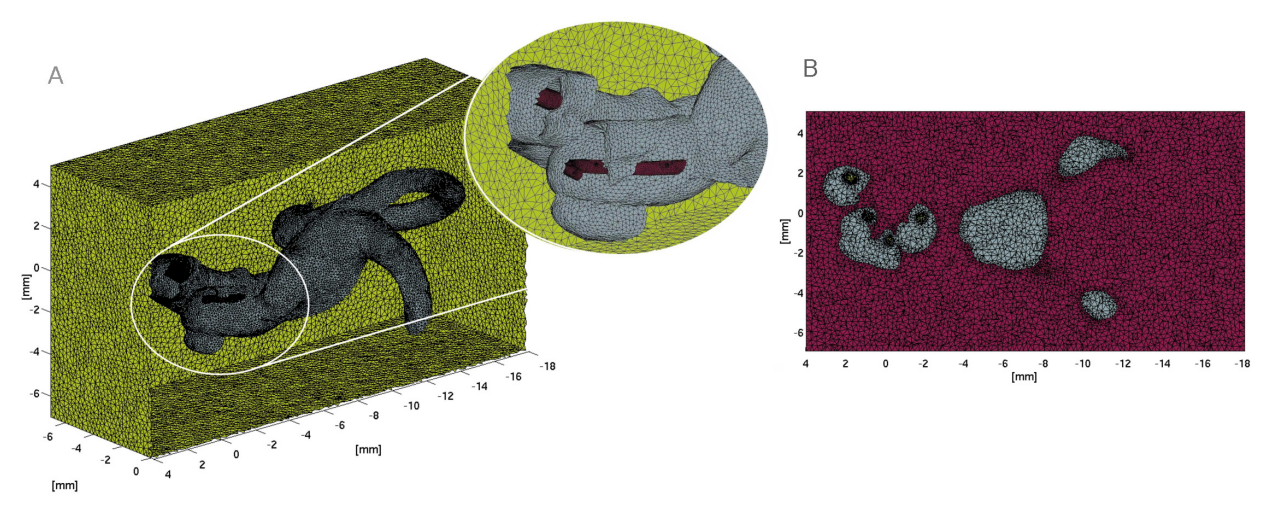

Figure 8: Final volumetric mesh. Cut of the obtained tetrahedral mesh, faces (A) and elements (B). The zoom focuses on the area where the electrode mesh is placed.

into account heterogeneous data as our framework does. Despite this, they present the following advantages: the possibility to adapt the framework to general applications [33] or to create either tetrahedral or hexahedral meshes [38]. In contrast with other studies [38], our work does not simplify the implant geometry, since its geometrical properties are relevant in further optimization steps.

A limitation of the proposed framework is the lack of realism of the bone mesh created around the cochlea, currently approximated by a box. Next improvements will be towards obtaining a bone structure reconstruction from clinical data [38]. Also, the electrode mesh elements are modified because of the geometrical deformation approach to carry out the virtual insertion. This may lead to instabilities in the FE simulation. Our results show that the change of element area after the deformation is low and does not lead to degenerate elements. Furthermore, the electrode length was not altered, keeping the depth insertion within reported ranges [10]. The position of the electrodes is in agreement with the literature $[12,10]$. We therefore conclude that our approach is promising for the purpose of the current work.

Considering the cochlea as a rigid body obviates the fact that the insertion may cause damage to the lateral wall and basilar membrane. However, we believe that this is a reasonable simplification given that our focus is to compute the final position of the implant to further create the computational mesh. The effect of the potential trauma over the overall electrical stimulation is minor, and not relevant for the type of planning that we are focusing on.

Tetrahedral elements may be of limited use depending on the physical problem to be solved in the FE analysis [18]. Hexahedral elements have more stability in 
mechanical simulations when, for example, bone structures $[29,30]$ or fluid pressure in the middle ear [39] are analyzed. However, our computational model will not be used for mechanical simulations, neither structural nor fluid dynamics, but only electrical ones. No limitations in FE electrical analysis have been reported using tetrahedral elements for CI electrical analysis [3, 5, 38].

Once generated, the patient-specific computational model is used as input for FE electrical simulations, providing a detailed evaluation of the outcomes of the CI $[5,24,6]$. This represents a step forward for testing in-silico, in a fast and automatic way, the potential outcomes of CI in a patient-specific case. In-silico personalized models have strong potential for preoperative planning. We believe that our modular framework could be adapted to generate anatomically rich and detailed functional models of patients undergoing other surgical procedures.

\section{Acknowledgments}

This research was partially funded by the European Union Seventh Frame Programme (FP7/2007-2013), grant agreement 304857, HEAR-EU project.

\section{References}

[1] Allard, J., Cotin, S., Faure, F., Bensoussan, P.-J., Poyer, F., Duriez, C., Delingette, H. and Grisoni, L. Sofa - an open source framework for medical simulation. In: Medicine Meets Virtual Reality (MMVR'15), 2007.

[2] Bogunovic, H., Pozo, J., Cardenes, R., Villa-Uriol, M., Blanc, R., Piotin, M. and Frangi, A. Automated landmarking and geometric characterization of the carotid siphon. Med Image Anal. 16:889-903, 2012.

[3] Briaire, J. J. and Frijns, J.H.M. 3D mesh generation to solve the electrical volume conduction problem in the implanted inner ear. Simulation Practice and Theory. 1-2:57-73, 2000.

[4] Bucki, M., Payan, Y., Cannard, F., Diot, B. and Vuillerme, N. Multi-modal framework for subject-specific finite element model generation aimed at pressure ulcer prevention." Comput Method Biomec. 16:147-8, 2013.

[5] Ceresa, M., Mangado, N., Dejea, H., Carranza, N., Mistrik, P., Kjer, H., Vera, S., Paulsen, R., and González Ballester, M. Patient-specific simulation of implant placement and function for cochlear implantation surgery planning. In: Medical Image Computing and Computer-Assisted Intervention, LNCS, 8674:49-56, 2014. 
[6] Ceresa, M., Mangado, N., Andrew, R.J. and González Ballester, M. Computational models for predicting outcomes of neuroprosthesis implantation: the case of cochlear implants. J Mol Neurobiol 52:2, 934-941. 2015

[7] Chen, B. K., Clark, G. M. and Jones, R. Evaluation of trajectories and contact pressures for the straight nucleus cochlear implant electrode array - A twodimensional application of finite element analysis. Med. Eng. Phys. 25:141-147, 2003.

[8] Cootes, T. F., and Taylor, C. J. Active Shape Models.Their training and application.Comput Vis Image Und, 61(1):38-59. 1995

[9] Duchateau, N., Mangado, N., Ceresa, M., Mistrik, P., Vera, S. and González Ballester, M. Virtual cochlear electrode insertion via parallel transport frame. In: Proceedings of International Symposium on Biomedical Imaging,13981401,2015 .

[10] Escudé, B., James, C., Deguine, O., Cochard, N., Eter, E. and Fraysse, B. The size of the cochlea and predictions of insertion depth angles for cochlear implant electrodes. Audiol Neurotol 11:27-33, 2006.

[11] Fang, Q. and Boas, D. Tetrahedral mesh generation from volumetric binary and gray-scale images. In: IEEE International Symposium on Biomedical Imaging, 2009.

[12] Finley, C. C., Holden, T. A., Holden, L. K., Whiting, B. R., Chole, R. A., Neely, G. J., Hullar, T. E., and Skinner, M. W. Role of electrode placement as a contributor to variability in cochlear implant outcomes. Otol. Neurotol. 29: 920-928, 2008

[13] Franke-Trieger, A., Jolly, C., Darbinjan, A., Zahnert, T. and Mürbe, D. Insertion depth angles of cochlear implant arrays with varying length: a temporal bone study. Otol. Neurotol. 35:58-63, 2014.

[14] Gani, M., Valentini, G., Sigrist, A., Kós, M.I. and Boëx, C. Implications of deep electrode insertion on cochlear implant fitting. J. Assoc. Res. Otolaryngol. 29:920-928, 2008

[15] Gomes, G. T., Cauter, S. V., Beule, M. D., Vigneron, L., Pattyn, C. and Audenaert, E. A. Biomedical Imaging and Computational Modeling in Biomechanics, Springer Netherlands, 2013.

[16] Green, K., Bhatt, Y., Mawman, D., O'driscoll, M., Saeed, S., Ramsden, R. and Green, M. Predictors of audiological outcome following cochlear implantation in adults. Cochlear Implants Int 8:1-11,2007. 
[17] Hanekom, T. Modelling encapsulation tissue around cochlear implant electrodes. Medical and Biological Engineering and Computing 1:47-55,2005.

[18] Hughes,T.J.R. The Finite Element Method: Linear Static and Dynamic Finite Element Analysis. Prentice-Hall ,1987.

[19] Kjer, H., Vera, S., Fagertun, J., González Ballester, M. and Paulsen, R. Predicting detailed inner ear anatomy from clinical pre-op CT. Int $J$ Comput Assist Radiol Surg. 10 (Suppl1) : S98-S99, 2015.

[20] Kjer, H., Fagertun, J., Vera, S., Gil, D., González Ballester, M. A. and Paulsen, R. R. Free-form image registration of human cochlear $\mu \mathrm{CT}$ data using skeleton similarity as anatomical prior. Pattern Recognition Letters. 000:1-7, 2015

[21] Kwon, G.-H. ,Chae, S.-W. and Lee, K.-J. Automatic generation of tetrahedral meshes from medical images. Comput. Struct. 81:765-775, 2003.

[22] Larrabide, I., Kim, M., Augsburger, L., Villa-Uriol, M., Rüfenacht, D., and Frangi, A. Fast virtual deployment of self-expandable stents: Method and in vitro evaluation for intracranial aneurysmal stenting. Med. Image Anal. 16:721-730, 2012.

[23] Lobos, C. and Rojas-Moraleda, R. From segmented medical images to surface and volume meshes, using existing tools and algorithms. In: International Conference on Adaptive Modeling and Simulation, 2013.

[24] Mangado, N., Ceresa, M., Duchateau, N., Dejea Velardo, H., Kjer, H., Paulsen, R., Vera, S., Mistrik, P., Herrero, J. and González Ballester, M. Automatic generation of a computational model for monopolar stimulation of cochlear implants. Int J Comput Assist Radiol Surg. 10 (Suppl1):S67-S68, 2015 .

[25] Mangado, N., Duchateau, N., Ceresa, M., Kjer, H., Vera, S., Mistrik, P., Herrero, J. and González Ballester, M. Patient-specific virtual insertion of electrode array for electrical simulations of cochlear implants. Int J Comput Assist Radiol Surg. 10 (Suppl1):S102-S103, 2015.

[26] Neal, M. L., and Kerckhoffs,R. Current progress in patient-specific modeling. Brief. Bioinform., 11:111-126, 2009.

[27] Nesme, M., Payan, Y., and Faure, F. Efficient, Physically Plausible Finite Elements. In Eurographics. 2005 
[28] Peiró, J., Formaggia, L., Gazzola, M., Radaelli, A. and Rigamonti,V. Shape reconstruction from medical images and quality mesh generation via implicit surfaces. Int. J. Numer. Meth. Fluids 53:1339-1360, 2007.

[29] Pfeiler, T. W., Lalush, D. S., an Loboa, E. G. Semiautomated finite element mesh generation methods for a long bone. Comput Meth Prog Bio 85(3):196-202. 2007

[30] Ramos, a., and Simões, J. a. Tetrahedral versus hexahedral finite elements in numerical modelling of the proximal femur. Medical Engineering and Physics, 28(9): 916-924. 2006

[31] Rattay, F. and Leao, R. Naves and Felix, H. A model of the electrically excited human cochlear neuron. II. Influence of the three-dimensional cochlear structure on neural excitability. Hearing Research 1-2:64-79, 2001.

[32] Russ, C., Hopf, R., Simon, H. S., Born, S., Hirsch, S. and Falk, V. Computational stent placement in trasncatheter aortic valve implantation. In: 6th International Symposium, ISBMS, 2014.

[33] Shepherd, J. F. and Johnson, C. R. Hexahedral mesh generation for biomedical models in SCIRun. Eng. Comput. 25:97-114, 2009.

[34] Shewchuk, J. R. What is a Good Linear Element? Interpolation , Conditioning, and Quality Measures. In: 11th International Meshing Roundtable, 2002 .

[35] Sun, W. , Martin, C. and Pham, T. Computational modeling of cardiac valve function and intervention. Annu. Rev. Biomed. Eng. 16:53-76, 2014.

[36] Tabor, G., Young, P. G., West, T. B. and Benattayallah, A. Mesh Construction from Medical Imaging for Multiphysics Simulation: Heat Transfer and Fluid Flow in Complex Geometries. Eng. Appl. Comp. Fluid. 1:126-135, 2014.

[37] Thomas Roland,J. J. Cochlear implant electrode insertion. Oper. tech. otolaryngol. - head neck surg. 16: 86-92, 2005.

[38] Tran, P., Sue, A., Wong, P., Li, Q. and Carter, P. Development of HEATHER for Cochlear Implant Stimulation Using a New Modeling Workflow. IEEE Trans. Biomed. Eng 62:728-735, 2015.

[39] Zhang, J. and Bhattacharyya, S. and Simaan, N. Model and parameter identification of friction during robotic insertion of cochlear-implant electrode arrays. In: IEEE Int. Conf. on Robotics and Automation, 2009. 